\title{
An Integrated Tool for Assessing the Demand Profile Flexibility
}

\author{
Juan Alvaro Fuentes Moreno, Angel Molina García, Student Member, IEEE, Antonio Gabaldón Marín, Member, IEEE, \\ Emilio Gómez Lázaro, and Carlos Alvarez Bel, Member, IEEE
}

\begin{abstract}
The purpose of this paper is to describe an integrated tool whose aim is to assess the residential demand profile flexibility through the control of space conditioning loads, mainly air conditioner and heat pump appliances. This assessment has been divided into two principal tasks-an estimation of the controllable load and a selection of the optimum load control strategy according to a target profile and a set of prefixed constraints. The tool also provides the aggregated load behavior, allowing a comparison between different load strategies.

This tool can be applied from the customer's and the utility's side. An application example to a real environment is also presented.
\end{abstract}

Index Terms-Direct load control, energy management, load identification, load modeling.

\section{INTRODUCTION}

$\mathbf{I}$ $\mathrm{N}$ THE last decade, electrical power systems have experienced a series of changes and pressures which have modified the operation and planning of their most complex level: the distribution of the electric power to their end-users. From those changes, we can highlight the deregulation of the power market, the increasing significance of the distributed generation, and the continuous rise of the demand of energy.

In an ideal electricity market, demand-side has the opportunity to participate and compete with supply-side, for example, through demand-side bidding (DSB) [1]. To achieve the establishment of this market, it is necessary to develop and improve tools to manage, forecast, and evaluate the possibilities to perform bidding in these scenarios. Nowadays, unfortunately, the full participation of the demand in the energy and reliability markets is the exception rather than the rule [2].

This market establishment process is also accompanied with both: the technological maturity of small-size power plants, which allows a decentralization of the supply; and the rapid penetration of combined power and heat cogeneration, which improves the economical effectiveness of the distributed energy resources.

With respect to the demand growth, in this decade, the European Union assumes that the requirement of electricity will in-

Manuscript received May 6, 2003. This work was supported by the Ministerio de Ciencia y Tecnología of Spain through Research Project DPI20012779-C02-01.

J. A. Fuentes Moreno, A. M. García, A. G. Marín, and E. G. Lázaro are with the Department of Electrical Engineering, University Politécnica de Cartagena, Cartagena 30202, Spain (e-mail: juanalvaro.fuentes@upct.es; angel.molina@upct.es; direccion@etsii.upct.es; emilio.gomez@upct.es).

C. Alvarez Bel is with the Institute of Energy Engineering of the University

Politécnica de Valencia, Valencia 46022, Spain (e-mail: calvarez@die.upv.es).

Digital Object Identifier 10.1109/TPWRS.2003.821446 crease more than any other primary energy resource. This pattern is due to the successful adoption of new electrotechnologies, as well as the emergence of new end-uses in the tertiary and domestic sectors. For example, the growth experienced in the electrical demand of residential users in Spain was up to 30\% in the 1997-2002 period.

Traditionally, demand-side management (DSM) has been considered an efficient tool from supply-side. Unfortunately, some barriers have arisen to the DSM policy implementation. The reasons for this decline in the application of DSM policies are the following:

- residential customers are resistant to change in general, mainly due to the comfort problems detected in previous load management experiences;

- energy efficiency is not a high priority, in short term, for medium and large customers - there are a number of issues that have a higher priority, for example competitiveness, productivity, or quality - and they are reluctant to the risk-(i.e., not only from economical point of view) - associated with the changes in technology;

- decrease of governmental and utility funds to promote DSM policies since the beginning of the deregulation process.

In spite of these barriers during the last years, we expect that the new market should offer a lot of interesting opportunities to look for customer demand flexibility based on legal, economical, and technological factors. For example, and from a legal point of view, in a deregulated system, the demand and supply sides compete on an equal footing and they should have a similar potential as tools for the system operator.

For system operation purposes, there is no theoretical reason as to why the load cannot provide some of the services-reliability, energy, or ancillary - traditionally supplied by utilities, and perhaps with a lower response time.

For small and medium residential and commercial customers, the new market is a unique opportunity to reduce their costs through the management of their energy demand, applying load management and/or energy storage policies to perform demand bids in energy, balancing, or ancillary markets. This management of customer demand could be both utility-driven, the traditional DSM, or customer-driven: the so-called demand-responsive policies, not considered yet for small users.

To profit these opportunities, small users-through a demand aggregator-and medium users need software tools, as the one presented in this paper, to evaluate how flexible their demand is, in order to correct or improve their bids in energy markets by an appropriate management of their energy storage loads-air con- 
ditioner, heat pumps, electrical thermal storage devices, .... The results presented in this paper assume an interesting tool for utilities, distributors and, of course, medium and small customers to achieve the maximum profitability in this new scenario. The data and results are focused on a university scenario, the Universidad Politécnica de Cartagena, a medium customer in the deregulated Spanish market, which could perform energy bids in the near future.

\section{Demand FleXIBILITY AsSESSMENT PROCESS}

The developed tool to evaluate the demand flexibility and estimate the aggregated load behavior is basically formed by two differenced tasks. The first one covers the load disaggregation and identification problem, being possible to deduce the controllable load percentage - due to space conditioning loads, mainly air conditioner and heat pump appliances-for each interval time $\Delta_{n}$. These values offer an initial estimation of the demand flexibility opportunities; since the larger the controllable load percentage is, the higher profile modifications could be achieved. These outputs are used as inputs of the second task. In this task, the main objective is to determine the load control strategy which, applied on the controllable loads, minimizes the quadratic distance between a prefixed target and the global demand profile. The developed algorithm also allows to predict how the initial profile can finally be modified, shifting energy demanded from peak to nonpeak periods according to several constraints. This assessment is completed by applying the control actions to elemental load models previously developed and, subsequently, deduce the controlled and uncontrolled aggregated behavior. In the light of these results, and taking into account the discomfort degree-indoor temperature alterations - suffered by the customer as a consequence of the dutycycle modifications, it is possible to modify both the target demand profile and the constraints, determining the new results. Therefore, a close-loop process can be defined, of which the final results and assessment will depend on the operator opinion and perspectives.

The global diagram is shown in Fig. 1, where the main tasks have been boxed with discontinuous lines. In this scheme, the methodologies applied in each step have also been indicated. The following two sections are, respectively, focused on describing each of the tasks previously presented: load identification, and solution and simulation of optimum load control strategies.

\section{Controllable Load Estimation Problem}

In order to evaluate the possibilities of applying a load control strategy, an initial load research study has to be done. This load research will provide the answer to the question of which is the percentage of the total load that can be controlled along a time period. As it is an evaluation, it is preferred a load research study that gives a quick answer to this question rather than a more exact study, but with greater time and money requirements.

With these requirements in mind, a bibliographical research, see [3], of the principal techniques for end-use monitoring was made, being the most advantageous for our purposes the

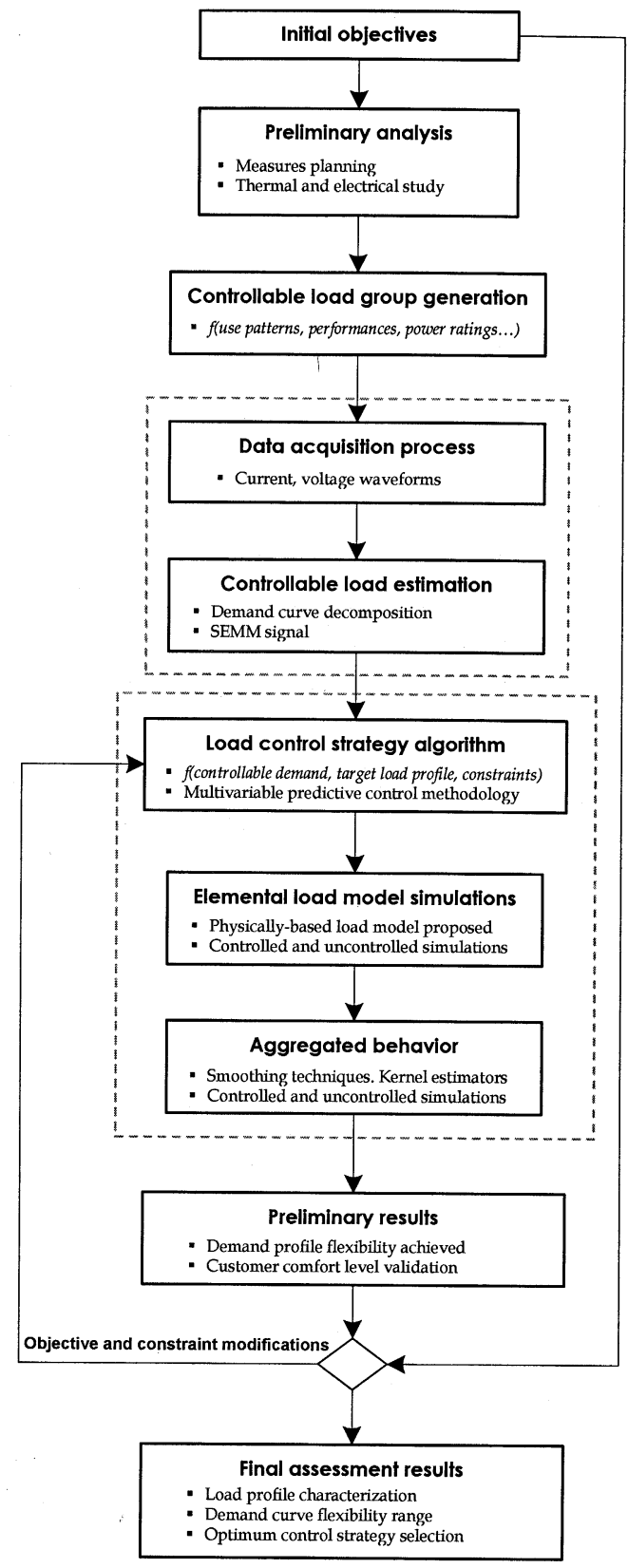

Fig. 1. Global tool scheme.

so-called nonintrusive techniques, which does not need access to customer loads in contrast with intrusive techniques.

These nonintrusive techniques simplify the hardware resources, using complex software that processes nonintrusive appliance unidirectional signatures. A signature is defined as any measurable parameter of the total load that gives information about the nature or operating state of an individual appliance in the load [4]

Non-intrusive load monitoring [4] is a nonintrusive technique designed to monitor, at a single point of an electrical circuit that supplies power to a number of devices, the desired load data using as load signature the step changes in the total measured power due to the connection or disconnection of the devices. As the aggregation level increases, this signature is more difficult to use for identification purposes, principally due to two problems: 
the number of power step changes increases, and the percentage of the power step, relative to the total load decreases. To overcome these difficulties, the solution is the use of other signatures that could be observed at higher aggregation levels.

As for our load research purposes, there is no need to identify the load absorbed by individual appliances, but an estimation of the total controllable load, two different signatures have been studied as signatures of a nonintrusive load monitoring technique that gives us an approximation of the controllable load. These signatures are harmonic frequency and subharmonic frequency signatures. The former signature is used in the technique entitled load decomposition, while the latter is used in a technique named SEMM signal. Both methods have in common that they analyze the current and voltage waveforms measured at a single point of the distribution network, so that they can give results of their predictions in real time.

\section{A. Load Decomposition Technique}

This technique is similar to the Fourier analysis of a waveform, decomposing the original waveform into a set of basic waveforms. The main difference between both methods is the set of waveforms used. In this way, while the Fourier analysis uses a set of sinusoidal waveforms, the load decomposition technique uses the current waveforms absorbed by individual loads, [5]. Thus, first we have to obtain, in the laboratory, individual electrical models of typical loads. Then, the current waveforms predicted by these models are used as the set of basic waveforms for the decomposition of the current measured at a certain point of the distribution network.

In [6], the process proposed to obtain the individual load models was the following: each device was supplied by a set of six different voltage levels, recording its input currents. Each voltage level was as distortion-free as possible, making the assumption that, for most devices, small distortions in the shape of the input voltage do not affect the current significantly. Finally, the Fourier components of the current amplitudes and phases up to the 30th harmonic were stored. In this methodology, the harmonic interaction between voltage and current harmonics of different order was neglected. In order to take into account this interaction, the ECAM model, described in [7], has been used in our nonintrusive load identification process [8]. This ECAM model is a black-box-type model that relates the supplied voltage harmonics with the absorbed current harmonics of the load through a matrix named enhanced crossed admittance matrix (ECAM)

$$
\bar{I}_{n \times 1}=[Y(\theta)]_{n \times m} \cdot \bar{V}_{m \times 1}
$$

where $\bar{V}_{m \times 1}$ is a vector formed by the fundamental component and its harmonics up to order $m$ of the supplied voltage; and $\bar{I}_{n \times 1}$ is a vector formed by the fundamental and the harmonics, up to order $n$ of the current absorbed by the load.

The ECAM matrix $[Y(\theta)]_{n \times m}$ depends on $\theta$, being $\theta$ the phase of the present voltage harmonic with respect to the fundamental component.

The results obtained in laboratory with this load decomposition process have shown that it is not possible to obtain reliable information about individual loads, despite modeling the

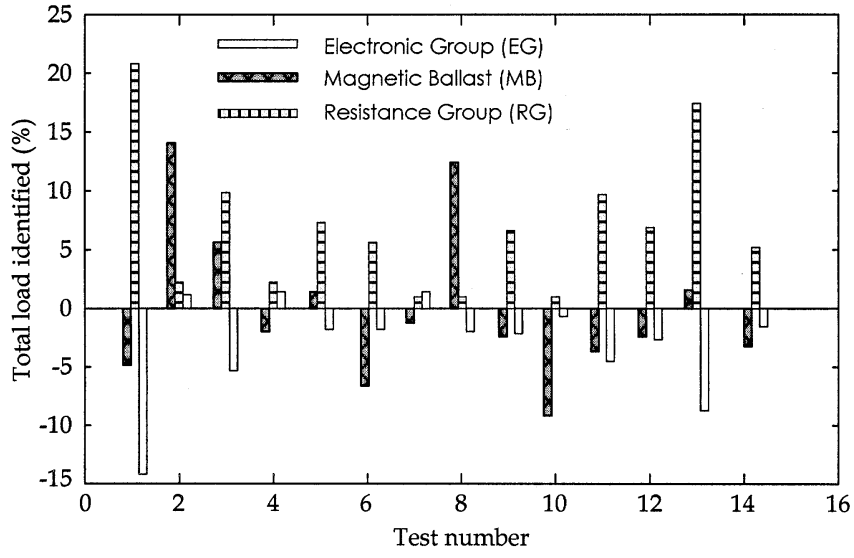

Fig. 2. Load group decomposition error.

harmonic interaction between voltage and current. However, if the initial decomposition of the measured current is finally aggregated into groups with similar electrical behavior, then the results obtained for each group could be used for obtaining information about the total load absorbed by each one Fig. 2 .

In our laboratory tests, three groups were made: a resistance group (RG), a magnetic ballast group (mainly fluorescent lamps) $[\mathrm{MB}]$, and the electronic group (mainly computers) [EG]. The resistance group includes the rest of the load that remains after the magnetic ballast group and the electronic group were identified. Therefore, it contains mostly motor and resistance loads.

For our identification purposes, the use of the load decomposition method will give an upper limit of the load absorbed by the air conditioner loads. It is an upper limit because it will also give us the power demanded by loads whose behavior (current absorbed) is similar to a resistance. The benefit of this method is that measuring at a single point of the distribution network we can obtain an estimation of the load absorbed by all of the controllable loads being supplied.

A distinct approximation to obtain an estimation of the controllable load could be the study of the SEMM signal, which is only related with motor loads.

\section{B. SEMM Signal Technique}

This technique analyzes the current subharmonic produced by the electrical loads that contain single phase motors, as could be, for example, air conditioner loads, heat pumps, refrigerators, etc. This subharmonic can be used as a nonintrusive signature that will give us information about these loads [4].

In order to study the suitability of this signal, measurements of the current absorbed by typical air conditioner loads were made. It was found that for all of the measured loads, the current absorbed by them presented a subharmonic whose frequency varied from 1 to $3 \mathrm{~Hz}$ (Fig. 3). As can be seen, the current presents a subharmonic oscillation in its maximum and minimum magnitudes. These maximum and minimum values, per cycle of such current, are separately plotted. In both cases, a ripple of around $0.5 \mathrm{~A}$ and $1 \mathrm{~Hz}$ oscillating over a constant value of $4 \mathrm{~A}$ can be found. This subharmonic signature could be improved if both curves were added, since the constant value disappears and the subharmonic amplitude is doubled. This last sig- 


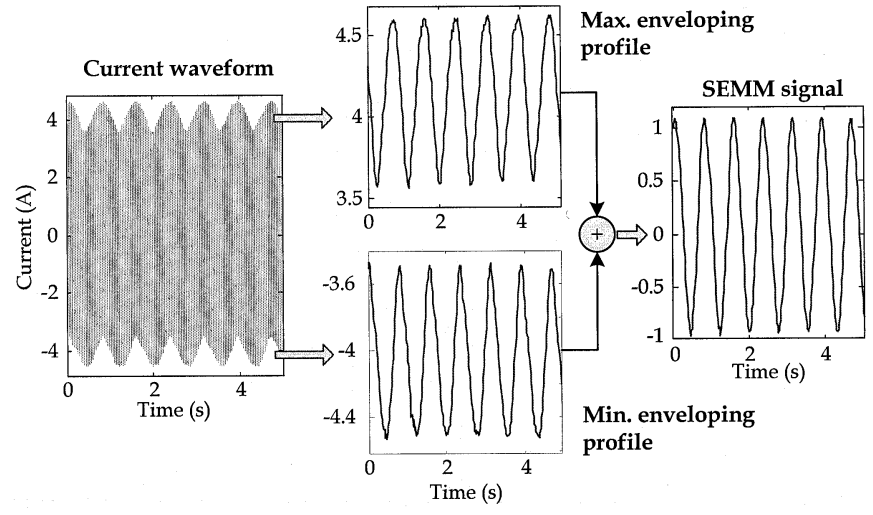

Fig. 3. SEMM signal deduction.

nature, obtained by adding the extreme values, for each cycle, is named SEMM signal.

Since the subharmonics of different devices are not synchronized in time, the total amplitude of the subharmonic current absorbed by an aggregation of such loads will present a cancellation effect similar to the harmonic currents, which is due to the phase-angle diversity of individual harmonics [9]. This amplitude cancellation behavior implies that as the aggregation level increases, the reliability of this SEMM signal decreases, which is due to the subharmonic magnitude reduction.

Despite this behavior, a Monte Carlo simulation has been made to investigate the combined effect of subharmonic cancellation due to an aggregation of air conditioner loads. For each simulation, corresponding to a specific load aggregation level, it has randomly been chosen, from the set of measured air conditioners, a current waveform that has also been randomly shifted in time to simulate a random load connection process. This process has been done as many times as needed until the simulated load equals the desired load aggregation level. To take into account the contribution of different air conditioner appliances with different subharmonic frequencies, it has been necessary to use as identification signature the RMS value of the simulated SEMM signal. The results obtained are shown in Fig. 4.

As seen in Fig. 4, when the RMS value of the SEMM signal increases, the interval of total air conditioner load identified also increases, but in greater proportion. This implies that, as expected, this method could only be used for identification purposes under low aggregation levels.

On the other hand, this information could be used as an alternative way to obtain information of the power demanded of the air conditioner load presented at a certain point of the distribution network. Besides, it can also be used to modulate the predicted values obtained using the previously introduced load decomposition technique.

\section{COntrol AND Simulation Problem}

\section{A. Elemental Load Modeling}

Traditionally, the aggregated power consumption of a residential load group has been obtained from the analysis of individual load behaviors. In this way, most authors have previously developed single load models, which allowed them to estimate

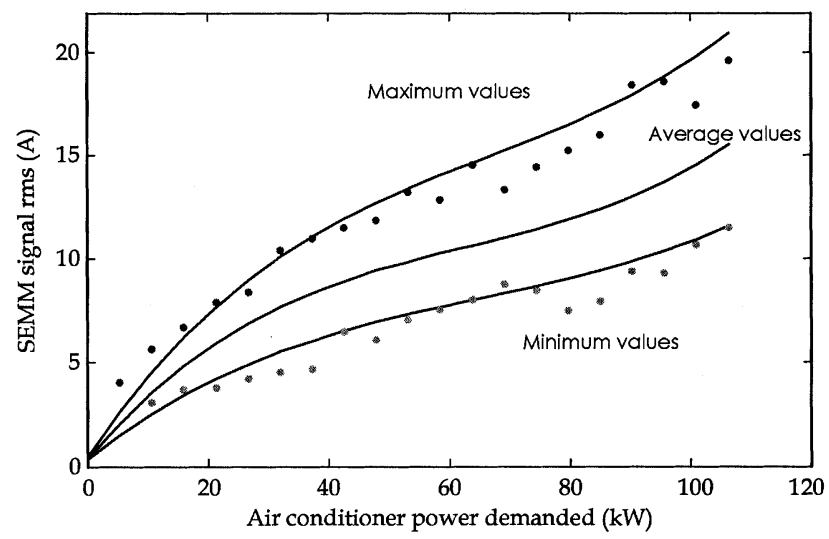

Fig. 4. SEMM signal. RMS characteristic.

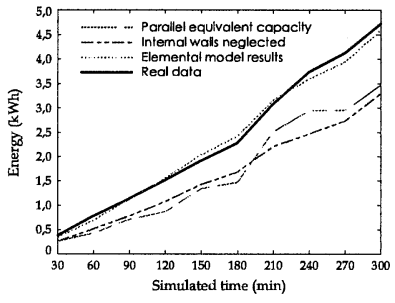

(a)

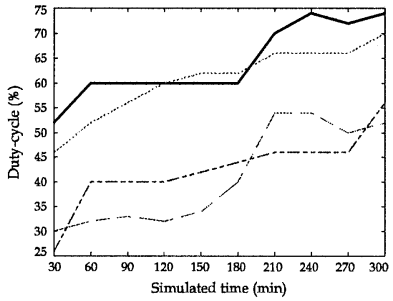

(b)
Fig. 5. Elemental load model. Comparison of results. (a) Energy comparison. (b) Duty-cycle comparison.

the individual demand profiles and, subsequently, derive the aggregated consumption.

During the last two decades, physically-based load modeling has been the most common methodology. The first application examples can be found in [10], [11]; whereas in [12], the stochastic aspect of the individual customer loads is also taken into account. Afterwards, [13] and [14] offer an improved but more general version of the previous load models.

In all cases, these models offer suitable consumption forecasts when the simulated time period is not very long, but present significant discrepancies if several hours are considered - which could be a typical implemented load control strategy period. For this reason, we have developed an improved third-order physically based elemental load model that takes into account parameters neglected in previous studies, and offers more accurate results when several hours are simulated [15].

In order to show the suitability of the developed elemental model in comparison with previous residential elemental load models, Fig. 5 presents a comparison between real data, the proposed elemental model, and two second-order models: in the first one, the internal wall thermal capacity has been neglected, and, in the second, an indoor equivalent thermal capacity, which takes into account the internal partitions and the indoor environment, has been considered. As seen in Fig. 5, the proposed model offers lower errors than the second-order simplified elemental models, with forecasted energy discrepancies around $30 \%$. In the case of the parallel equivalent capacity model, it gives results closer to the real data. However, its thermal and electrical elemental behavior still continue substantially far from the real performance (see Fig. 6). Therefore, 


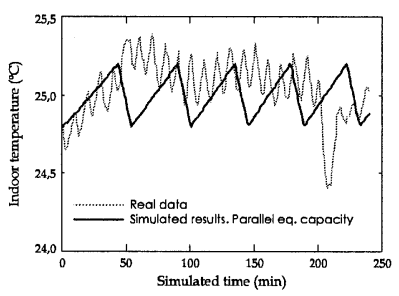

(a)

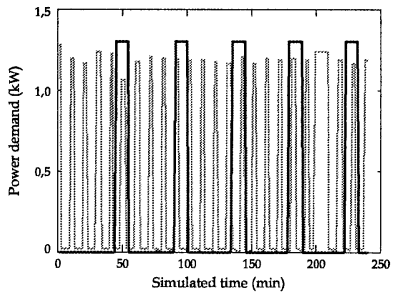

(b)
Fig. 6. Simplified elemental load model. (a) Indoor temperature. (b) Electrical power.

we propose to apply our developed third-order elemental load model to avoid these deficiencies and simulate correctly the electrical and thermal behavior.

\section{B. Aggregated Behavior of Elemental Loads}

Different aggregation methods have been developed as an answer to specific problems in electrical power systems, showing a parallel development together with the elemental load models. Thus, the first contributions proposed a simple addition of elemental loads, improving this initial approach by running a residential simulation elemental model for a long duration (e.g., a typical summer) and later estimating the probability-distribution to give the joint probability of an air conditioner appliance being ON for given input parameters [16]. In [17], one of the first attempts to introduce stochastic processes for aggregation purposes can be found, based on Markov chains. Later, several authors proposed to assemble the houses into different classes; such as in [13] a classification is suggested according to the air conditioner ratings, and [12] presents a methodology based on an elemental load aggregation into a group called homogeneous group by a similarity of the functional as well as electrical behavior. During the last decade, the aggregation problem was mainly solved by means of describing the appliance state dynamics-ON, OFF_-through the interaction of two coupled Fokker-Planck partial differential equation [14], [18]; solution previously proposed by Malhamé in [19] and applied in this case as homogeneous as no-homogeneous elemental load groups. Recently, the aggregation problem has been considered by defining a limit case, consisting in the aggregation of an infinite number of structurally identical infinitesimal loads with a given total mean power. Obviously, the limit case would be an infinite number of identical loads with a total constant power demand. Finally, one of the most recent works written about the residential load behavior has been again focused on cold load pick-up. In this case, a delayed exponential model has been suggested to simulate the aggregate demand, where the power consumption is initially constant, and then decreases exponentially toward a stationary level [20].

From our point of view, these methodologies have usually offered a partial characterization about the aggregated behavior-by means of assuming average values for dutycycle and elemental demands. Besides, the solution based on Fokker-Planck, one of the most widely used, presents an increasing mathematical complexity when the model parameters are considered as random variables or the number of
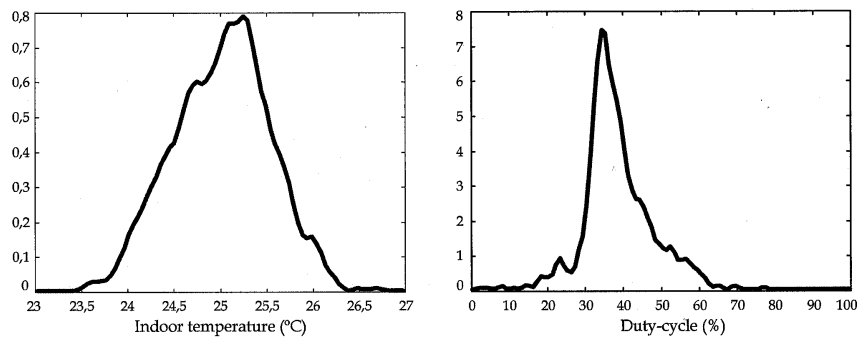

Fig. 7. Smoothing techniques. Example.

those parameters is increased, since the elemental load model expressions are explicitly considered in the coupled partial differential equations. In order to solve these deficiencies, we propose to define a nonparametric estimate of the marginal density $x \mapsto f(t ; x)$ of $x_{i n}(t)$ at any desired time $t$, based on the simulation of $N$ independent trajectories up to time $T$. The estimator, for a given time $t$, at $x$, is the kernel density estimator, see, for example, [21] for an account of the subject.

Fig. 7 shows the results obtained for an elemental load group, in which the thermal parameters have been modeled as random variables according to their nature and typical range, using the proposed elemental load model [22].

\section{Algorithms of Load Control Strategies}

The development of algorithms to control space conditioning residential loads have traditionally been related to demand-side management, specifically in load management programs. One of the first objectives was to decrease the generation costs, in this way, enumeration methods were initially applied to evaluate a sequence of candidate control schedules and, then, select the control schedule giving the lowest cost [23]. Afterwards, the objectives also included operational cost savings, as well as load peak decreasing, [24]. In order to achieve these objectives, different methodologies were implemented, such as dynamic programming [25]-[27] and linear programming [28], [29]. During the last years, alternative methodologies have been proposed with similar objectives; examples can be found in [30] and [31], which applied fuzzy logic and fuzzy dynamic programming, respectively, to reduce system peak loads and system operation costs. On the other hand, [32] proposed to schedule ON/OFF switching of residential appliances in accordance with a predefined load reduction profile through the pulse width modulation (PWM) methodology. Its main disadvantage is that the load reduction target curve was related to the interruptible load group - partial load demand, and not to the global demand profile. Finally, more recent authors have presented decision tools to select load control strategies in a multiobjective framework [33]. Nevertheless, these approaches start from a predefined strategy set-considered as input variables - and, therefore, it is assumed the control strategy selection process as a previous step which is necessary to solve.

Therefore, and taking into account the works previously presented, an algorithm to generate load control strategies has been developed in order to control optimally the space conditioning residential loads and modify the global demand curve profile according to a specific target load curve previously prefixed. This 
point of view allows us to apply the algorithm as utility-side as customer-side, assessing how flexible the global demand profile is. Constrained multivariable predictive control methodology has been used, avoiding typical load control problems as payback effect and shifting the demand from peak to nonpeak periods [34].

\section{ASSESSMENT EXAMPLE}

The proposed methodology is illustrated in this section for a system study. The system is located in an university area, formed by three department and lecture room buildings, with the total system peak demand being $450 \mathrm{~kW}$. In this example, the controllable load has been divided into three groups, where each group is assumed to have 75 devices ranging from 1 to 5 $\mathrm{kW}$. The conditioning elemental spaces present a relevant diversity of their thermal and electrical parameters: from 10 to $100 \mathrm{~m}^{2}$ floor space, external wall orientations uniformly distributed, from 10 to $35 \%$ of window surfaces, three-phase and single-phase appliances, etc. To take into account all of this variability, the elemental load model parameters have been characterized by means of random variables, selecting the suitable density function profiles according to the modeled parameter nature.

\section{A. Load Identification: The Demand Disaggregation}

The demand disaggregation is the first task that has to be done and its final result is a measurement of the controllable load percent due to space conditioning loads in the university area.

A preliminary analysis of the typical loads present in its facilities was done. And it was found that the principal loads were: air conditioner loads, lighting - mainly magnetic ballast with fluorescent tubes, and computer loads.

In this study case, there was no possible confusion about the loads that were present in the resistive group-mainly air conditioner, and this facilitated the use of the load decomposition nonintrusive method, which simplifies the measurement process because it can be used at higher aggregation levels than the SEMM signal technique. In this way, it was only considered necessary the measurement of the current and voltage waveforms at the distribution transformer of the three buildings.

In order to make the waveforms acquisition process, a specially developed software was implemented that took the require voltage and current data, for each phase, at 5-min intervals and with the necessary speed of sampling to perform the decomposition process. Previously, a database of model loads had to be created, measuring in laboratory the current absorbed by each of the loads when they were supplied with different voltage waveforms generated with an arbitrary waveform generator, thus obtaining their ECAM load model.

With the acquired voltage and current waveforms-measured at the distribution transformer-and the available load models-measured in laboratory-the load decomposition technique was applied. The final results of the decomposition process are shown in Fig. 8, where the curves represent the load absorbed by each of the basic load groups.

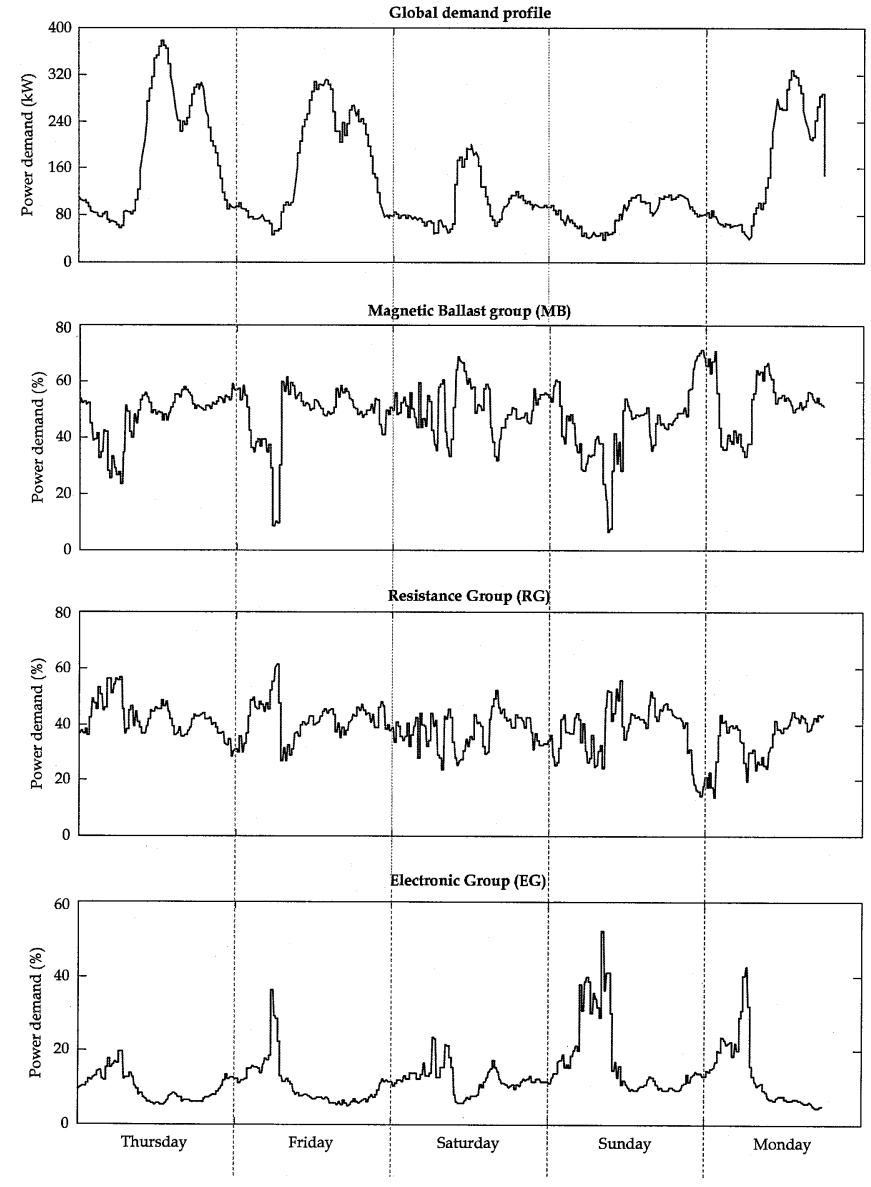

Fig. 8. Load identification. Demand disaggregation.

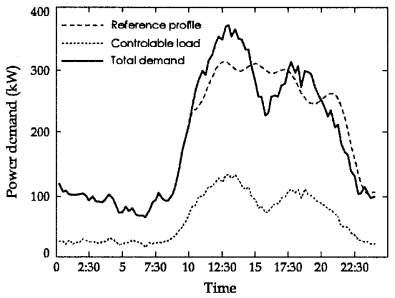

(a)

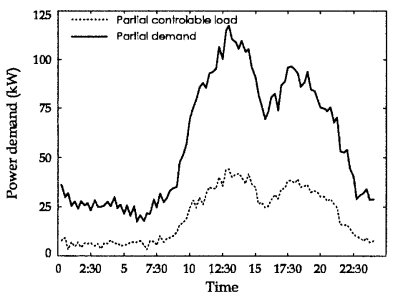

(b)
Fig. 9. Initial demand profiles. Global reference curve. (a) Global demand profiles. (b) Partial demand profiles.

\section{B. Control Strategies: Demand Flexibility Range}

Once the disaggregation problem has been solved, it is possible to assess how flexible the demand profile is. A typical day period has been selected and a target reference profile has been defined according to the desired modifications. Fig. 9 shows the global demand profiles as well as the partial demand corresponding to a specific customer group, taking into account the results derived from the load identification process.

The load control algorithm, according to the input parameters and the prefixed constraints, gives a control action vector for each customer group. These values represent the relationship, for each 15-min interval time, between the energy demanded with control and the energy absorbed by the load group if these elemental loads were uncontrolled. Fig. 10 shows the obtained 


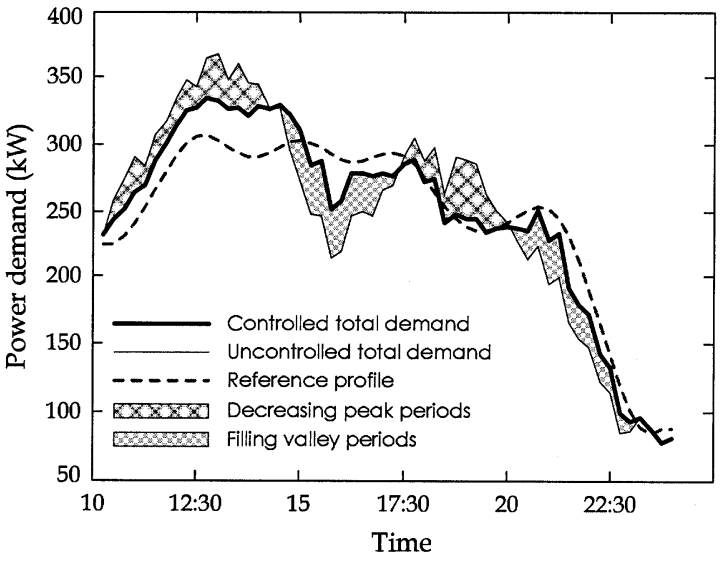

Fig. 10. Controlled demand profile. Flexibility assessment.

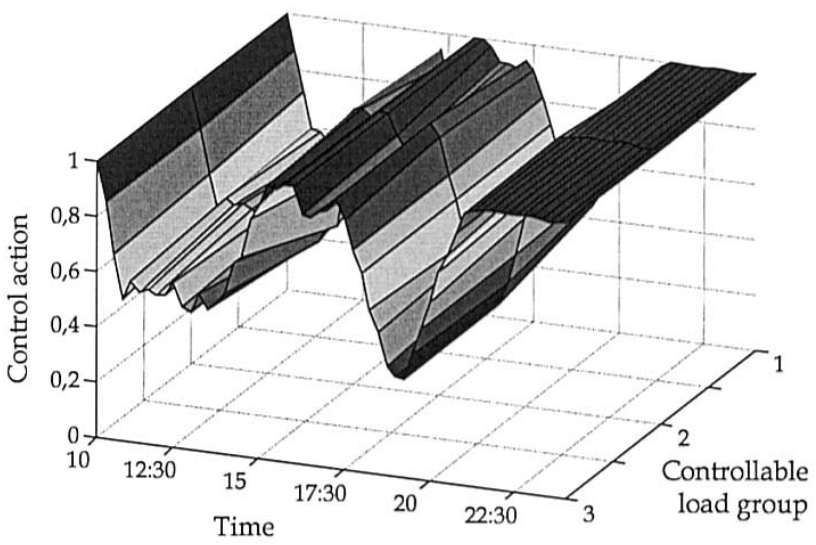

Fig. 11. Control actions.
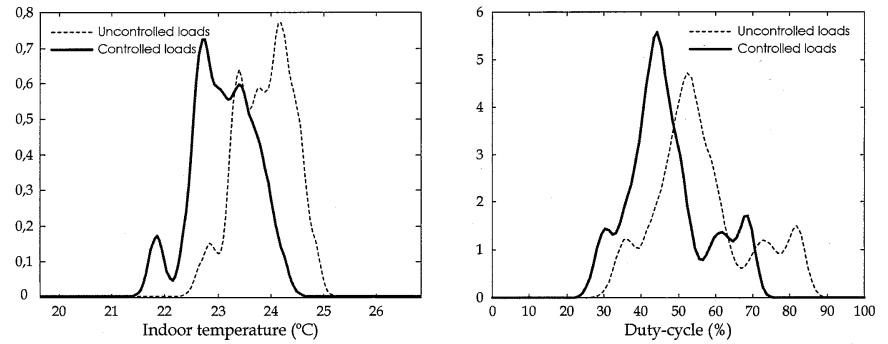

Fig. 12. Aggregated behavior. Controlled load group.

results and the final demand profile when the control actions (Fig. 11) are implemented.

In order to evaluate the customer comfort level under this kind of action, the proposed elemental load model and smoothing techniques have been used to know the aggregated behavior. In this way, three space conditioning residential load groups have been simulated according to the characteristics of the studied system. Fig. 12 shows the density function profiles corresponding to one of these load groups when their control actions are implemented, comparing these results with its natural behavior. These curves have been determined, in both cases, for a simulated time of $120 \mathrm{~min}$, which correspond to a consumption reduction period-control actions below 1 . In the same way, this kind of information can be useful to know how much the indoor temperature has been modified, and achieve an additional viewpoint in reference to a specific control strategy, allowing the introduction of constraint modifications and testing more flexible demand profiles.

\section{CONCLUSION}

A tool to assess the demand profile flexibility by means of controlling space conditioning residential loads is presented. The developed environment solves two interrelated objectives: the controllable load identification problem, and the selection and simulation of an optimum load control strategy according to a prefixed target demand profile. The former has been formulated by means of a nonintrusive methodology_load decomposition technique - that uses the current and voltage waveforms measured at a point of a radial distribution network; the latter is solved through the use of multivariable constraint predictive control methodology. The simulation of the controllable load groups is made by aggregating elemental load models through an aggregation methodology previously proposed by the authors.

As main advantages of this integrated tool, in comparison with previous approaches, the following can be pointed out: individual load monitoring is not necessary to estimate the controllable load; shaping global demand according to a target profile can be obtained; and the aggregation process is not associated with the mathematical expressions of the elemental loads, but to their individual simulation results.

The tool could be applied from both the utility's and the customer's point of view. An application of the proposed tool is also presented in order to test it in a real environment.

\section{REFERENCES}

[1] I. E. Agency, "Task VIII. Demand Side Bidding in a Competitive Electricity Market," Demand-Side Management International Energy Agency Tech. Rep., 2001.

[2] E. Hirst, "Reliability benefits of price-responsive demand," IEEE Power Eng. Rev., vol. 22, 2002.

[3] A. T. Almeida and E. L. Vine, "Advanced monitoring technologies for the evaluation of demand-side management programs," IEEE Trans. Power Syst., vol. 9, pp. 1691-1697, Aug. 1994.

[4] G. W. Hart, "Nonintrusive appliance load monitoring," Proc. IEEE, vol. 80, no. 12, pp. 1870-1891, Dec. 1992.

[5] H. Yan, D. Czarkowski, L. Birembaum, E. Levi, and J. Hajagos, "Experimental test of a load model in the presence of harmonics," Proc. Inst. Elect. Eng., Gen. Transm. Dist., vol. 146, no. 2, pp. 186-192, Mar. 1999.

[6] R. Mancini, Z. Zabar, L. Birembaum, E. Levi, J. Hajagos, and S. Kalinowsky, "An area substation load model in the presence of harmonics," IEEE Trans. Power Delivery, vol. 11, pp. 2013-2019, Oct. 1996.

[7] J. Fuentes, A. Gabaldón, F. Cánovas, and A. Molina, "Harmonic model of electronically controlled loads," in IEEE Power Eng. Soc. Summer Meet., vol. 3, July 2000, pp. 1805-1810.

[8] J. Fuentes, A. Gabaldón, E. Gómez, A. Molina, and F. Ruz, "Object oriented architecture of a load decomposition identification system at distribution level," in Proc. 14th Power Syst. Comput. Conf., June 2002.

[9] A. Mansoor and W. M. Grady, "Analysis of compensation factor influencing the net harmonic current produced by single-phase nonlinear loads," in Proc. 8th Int. Conf. Harmon. Quality Power, Oct. 1998, pp. 883-889.

[10] S. Ihara and F. C. Schweppe, "Physically based modeling of cold load pickup," IEEE Trans. Power App. Syst., vol. 100, pp. 4142-4150, Sept. 1981.

[11] T. Calloway and C. Brice, "Physically-based model of demand with applications to load management assessment and load forecasting," IEEE Trans. Power App. Syst., vol. PAS-101, pp. 4625-4630, Dec. 1982.

[12] C. Y. Chong and R. Malhamé, "Statistical synthesis of physically based load models with applications to cold load pickup," IEEE Trans. Power App. Syst., vol. PAS-103, pp. 1612-1628, July 1984. 
[13] A. Pahwa and C. W. Brice, "Modeling and system identification of residential air conditioning load," IEEE Trans. Power App. Syst., vol. PAS-104, pp. 1418-1425, June 1985.

[14] C. Alvarez, R. Malhamé, and A. Gabaldón, "A class of models for load management application and evaluation revisited," IEEE Trans. Power Syst., vol. 7, pp. 1435-1443, Nov. 1992.

[15] A. Molina, A. Gabaldón, J. A. Fuentes, and C. Álvarez, "Implementation and assessment of physically based electrical load models: application to direct load control residential programmes," Proc. Inst. Elect. Eng., Gen. Transm. Dist., vol. 150, no. 1, pp. 61-66, Jan. 2003.

[16] M. L. Chan and G. B. Ackerman, "Simulation-based load synthesis methodology for evaluating load-management programs," IEEE Trans. Power App. Syst., vol. PAS-100, pp. 1771-1778, Apr. 1981.

[17] H.-T. Nguyen, J. D. Birdwell, and F. Hall, "A physically-based low-order model for aggregate air conditioner loads," in Proc. Amer. Contr. Conf., June 1982, pp. 1152-1154.

[18] J. C. Laurent and R. P. Malhamé, "A physically-based computer model of aggregate electric water heating loads," IEEE Trans. Power Syst., vol. 9, pp. 1209-1217, Aug. 1994

[19] R. P. Malhamé and C. Y. Chong, "Electric load model synthesis by diffusion approximation of a high-order hybrid-state stochastic system," IEEE Trans. Automat. Contr., vol. AC-30, pp. 854-860, Sept. 1985.

[20] E. Agneholm and J. Daalder, "Cold load pick-up of residential load," Proc. Inst. Elect. Eng., Gen. Transm. Dist., vol. 171, no. 1, pp. 44-50, 2000.

[21] W. Hrdle, Smoothing Techniques: With Implementation in S. New York: Springer-Verlag, 1991.

[22] A. Molina, A. Gabaldón, M. Kessler, and J. A. Fuentes, "Application of smoothing techniques to solve the cooling and heating residential load aggregation problem," in Proc. VII Int. Conf. Probabilistic Methods Appl. to Power Syst., vol. 2, 2002, pp. 879-886.

[23] R. Bhatnagar and S. Rahman, "Dispatch of direct load control for fuel cost minimization," IEEE Trans. Power Syst., vol. PWRS-1, pp. 96-102, Nov. 1986.

[24] J. Chen, F. N. Lee, A. M. Breipohl, and R. Adapa, "Scheduling direct load control to minimize system operational cost," IEEE Trans. Power Syst., vol. 10, pp. 1994-2001, Nov. 1995.

[25] A. I. Cohen and C. C. Wang, "An optimization method for load management scheduling," IEEE Trans. Power Syst., vol. 3, pp. 612-618, May 1988.

[26] Y.-Y. Hsu and C.-C. Su, "Dispatch of direct load control using dynamic programming," IEEE Trans. Power Syst., vol. 6, pp. 1056-1061, Aug. 1991.

[27] D.-C. Wei and N. Chen, "Air conditioner direct load control by multi-pass dynamic programming," IEEE Trans. Power Syst., vol. 10, pp. 307-313, Feb. 1995.

[28] C. N. Kurucz, D. Brandt, and S. Sim, "A linear programming model for reducing system peak through customer load control programs," IEEE Trans. Power Syst., vol. 11, pp. 1817-1824, Nov. 1996.

[29] K.-H. Ng and G. B. Sheblé, "Direct load control—a profit-based load management using linear programming," IEEE Trans. Power Syst., vol. 13, pp. 688-695, May 1998.

[30] K. Bhattacharyya and M. L. Crow, "A fuzzy logic based approach to direct load control," IEEE Trans. Power Syst., vol. 11, pp. 708-714, May 1996.

[31] H.-T. Yang and K.-Y. Huang, "Direct load control using fuzzy dynamic programming," Proc. Inst. Elect. Eng., Gen. Transm. Dist., vol. 146, no. 3, pp. 294-300, May 1999.

[32] N. Navid-Azarbaijani and M. H. Banakar, "Realizing load reduction functions by aperiodic switching of load groups," IEEE Trans. Power Syst., vol. 11, pp. 721-727, May 1996.

[33] H. Jorge, C. H. Antunes, and A. G. Martins, "A multiple objective decision support model for the selection of remote load control strategies," IEEE Trans. Power Syst., vol. 15, pp. 865-872, May 2000.
[34] A. Molina, A. Gabaldón, J. A. Fuentes, and F. J. Cánovas, “Approach to multivariable predictive control applications in residential HVAC direct load control," in Proc. IEEE Summer Meeting, July 2000.

Juan Alvaro Fuentes Moreno received the electrical engineering degree from the University of Zaragoza, Zaragoza, Spain, in 1994, and the Ph.D. degree from the University Politécnica de Cartagena, Cartagena, Spain, in 2001.

Currently, he is with the Department of Electrical Engineering at the University Politécnica de Cartagena. His research interests include load modeling, control, and optimization.

Angel Molina García (S’01) received the electrical engineering degree from the University Politécnica de Valencia, Valencia, Spain, in 1998, and the Ph.D degree from the University Politécnica de Cartagena, Cartagena, Spain, in 2003.

Currently, he is with the Department of Electrical Engineering at the University Politécnica de Cartagena, Cartagena, Spain. His research interests include demand-side bidding, load modeling, theory, and direct load control applications.

Antonio Gabaldón Marín (M'96) received the electrical engineering and Ph.D degrees from the University Politécnica de Valencia, Valencia, Spain, in 1988 and 1991, respectively. Currently, he is with the Department of Electrical Engineering at the University Politécnica de Cartagena, Cartagena, Spain.

His research interests include demand-side management, demand-side bidding, end-use efficiency, load modeling, and distribution automation.

Emilio Gómez Lázaro received the M.Sc. and Ph.D. degrees in electrical engineering from the University Politécnica de Valencia, Valencia, Spain, in 1995 and 2000, respectively.

Currently, he is an Associate Professor of Electrical Engineering at the University Politécnica de Cartagena, Cartagena, Spain. His current research interests include numerical methods for electromagnetism, large-scale parallel computing, scientific computing and engineering, load modeling, and control.

Carlos Alvarez Bel (M'80) received the electrical engineering and Ph.D. degrees from the University Politécnica de Valencia, Valencia, Spain, in 1976 and 1979 , respectively.

Currently, he is Professor in the Department of Electrical Engineering at the University Politécnica de Valencia. His research interests include demand-side management, distribution automation, and electric markets. 